\title{
Domain theory and general relativity
}

Keye Martin and Prakash Panangaden

Naval Research Laboratory

Center for High Assurance Computer Systems

Washington DC 20375

keye.martin@nrl.navy.mil

School of Computer Science

McGill University

Montreal, Quebec H3A 2A7

Canada

prakash@cs.mcgill.ca

Summary. We discuss the current state of investigations into the domain theoretic structure of spacetime, including recent developments which explain the connection between measurement, the Newtonian concept of time and the Lorentz distance.

\section{Introduction}

Domains [AJ94, GKK] are special types of posets that have played an important role in theoretical computer science since the late 1960s when they were discovered by Dana Scott [Sco70] for the purpose of providing a semantics for the lambda calculus. They are partially ordered sets that carry intrinsic (order theoretic) notions of completeness and approximation. The basic intuition is that the order relation captures the idea of approximation qualitatively. There is an abstract notion of finite piece of information, or of finite approximation, which plays a key role in the analysis of computation.

These posets have a number of topologies defined on them: the Scott topology and the interval topology, in particular. The Scott topology is particularly important in that continuity with respect to this topology captures some of the information processing aspects of computability. In particular, a Scott continuous function has the following property: a finite piece of information about the output requires only a finite piece of information about the input. While this does not completely reduce Turing computability to topology it captures a very crucial information processing aspect of computable functions.

General relativity is Einstein's theory of gravity in which gravity is understood not in terms of mysterious "universal" forces but rather as part of the geometry of spacetime. It is profoundly beautiful and beautifully profound 
from both the physical and mathematical viewpoints and it teaches us clear lessons about the universe in which we live that are easily explainable. For example, it offers a wonderful explanation of gravity: if an apple falls from a tree, the path it takes is not determined by the Newtonian ideal of an "invisible force" but instead by the curvature of the space in which the apple resides: gravity is the curvature of spacetime. In addition, the presence of matter in spacetime causes it to "bend" and Einstein even gives us an equation that relates the curvature of spacetime to the matter present within it.

The study of spacetime structure from an abstract viewpoint - i.e., not from the viewpoint of solving differential equations - was initiated by Penrose [Pen65] in a dramatic paper in which he showed a fundamental inconsistency of gravity. It was known since Chandrasekhar [Cha31] that since everything attracts everything else a gravitating mass of sufficient size will eventually collapse. What Penrose showed was that any such collapse eventually leads to a singularity where the mathematical description of spacetime as a continuum breaks down. This leads to the need to reformulate gravity. It is hoped that the elusive quantum theory of gravity will resolve this problem.

Since the first singularity theorems [Pen65, HE73] causality has played a key role in understanding spacetime structure. The analysis of causal structure relies heavily on techniques of differential topology [Pen72]. For the past decade Sorkin and others [Sor91] have pursued a program for quantization of gravity based on causal structure. In this approach the causal relation is regarded as the fundamental ingredient and the topology and geometry are secondary.

In a paper that appeared in 2006 [KP], we prove that the causality relation is much more than a relation - it turns a globally hyperbolic spacetime into what is known as a bicontinuous poset. The order on a bicontinuous poset allows one to define an intrinsic topology called the interval topology. On a globally hyperbolic spacetime, the interval topology is the manifold topology. Theorems that reconstruct the spacetime topology have been known [Pen72] and Malament [Mal77] has shown that the class of time-like curves determines the causal structure. We establish these results as well though in a purely order theoretic fashion: there is no need to know what "smooth curve" means.

Our more abstract stance also teaches us something new: a globally hyperbolic spacetime itself can be reconstructed in a purely order theoretic manner, beginning from only a countable dense set of events and the causality relation. The ultimate reason for this is that the category of globally hyperbolic posets, which contains the globally hyperbolic spacetimes, is equivalent to a very special category of posets called interval domains. This provides a profound connection between domain theory, first introduced for the purposes of assigning semantics to programming languages, and general relativity, a theory meant to explain gravity. Even from a purely mathematical perspective this equivalence is surprising, since globally hyperbolic spacetimes are usually not order theoretically complete, but interval domains always are. 
Measurements were introduced by Martin in [Mar00a]. One thing they provide is a way of incorporating quantitative information into domain theory. More recently we have also shown how the geometry of spacetime can be reconstructed order theoretically. The reason is that the Lorentz distance defines a Scott continuous function on the domain of spacetime intervals. What is even more interesting, though, is that our setting provides a way to topologically distinguish between Newtonian and relativistic notions of time. Every global time function defines a measurement on the domain of spacetime intervals, in particular, it is Scott continuous. The Lorentz distance is not only Scott continuous, but satisfies a stronger property, that it is interval continuous. An interval continuous function must assign zero to any element which approximates nothing. Thus, no interval continuous function on the domain of spacetime intervals can ever be a measurement and the reason for this has entirely to do with relativity: a clock moving at the speed of light records no time as having elapsed, so an interval continuous function is incapable of distinguishing between a single event and a null interval.

\section{Domains, continuous posets and topology}

A poset is a partially ordered set, i.e., a set together with a reflexive, antisymmetric and transitive relation.

Definition 1. Let $(P, \sqsubseteq)$ be a partially ordered set. A nonempty subset $S \subseteq P$ is directed if $(\forall x, y \in S)(\exists z \in S) x, y \sqsubseteq z$. The supremum of $S \subseteq P$ is the least of all its upper bounds provided it exists. This is written $\bigsqcup S$.

These ideas have duals that will be important to us: a nonempty $S \subseteq P$ is filtered if $(\forall x, y \in S)(\exists z \in S) z \sqsubseteq x, y$. The infimum $\bigwedge S$ of $S \subseteq P$ is the greatest of all its lower bounds provided it exists.

Definition 2. For a subset $X$ of a poset $P$, set

$$
\uparrow X:=\{y \in P:(\exists x \in X) x \sqsubseteq y\} \quad \& \downarrow X:=\{y \in P:(\exists x \in X) y \sqsubseteq x\} .
$$

We write $\uparrow x=\uparrow\{x\}$ and $\downarrow x=\downarrow\{x\}$ for elements $x \in X$.

A partial order allows for the derivation of several intrinsically defined topologies. Here is our first example.

Definition 3. A subset $U$ of a poset $P$ is Scott open if

(i) $U$ is an upper set: $x \in U \& x \sqsubseteq y \Rightarrow y \in U$, and

(ii) $U$ is inaccessible by directed suprema: For every directed $S \subseteq P$ with a supremum,

$$
\bigsqcup S \in U \Rightarrow S \cap U \neq \emptyset .
$$

The collection of all Scott open sets on $P$ is called the Scott topology. 
Posets can have a variety of completeness properties. The following completeness condition has turned out to be particularly useful in applications.

Definition 4. A dcpo is a poset in which every directed subset has a supremum. The least element in a poset, when it exists, is the unique element $\perp$ with $\perp \sqsubseteq x$ for all $x$.

The set of maximal elements in a dcpo $D$ is

$$
\max (D):=\{x \in D: \uparrow x=\{x\}\} .
$$

Each element in a dcpo has a maximal element above it.

Definition 5. For elements $x, y$ of a poset, write $x \ll y$ iff for all directed sets $S$ with a supremum,

$$
y \sqsubseteq \bigsqcup S \Rightarrow(\exists s \in S) x \sqsubseteq s .
$$

We set $\downarrow x=\{a \in D: a \ll x\}$ and $\uparrow x=\{a \in D: x \ll a\}$.

For the symbol "«," read "approximates."

Definition 6. A basis for a poset $D$ is a subset $B$ such that $B \cap \downarrow x$ contains a directed set with supremum $x$ for all $x \in D$. A poset is continuous if it has a basis. A poset is $\omega$-continuous if it has a countable basis.

Continuous posets have an important property, they are interpolative.

Proposition 1. If $x \ll y$ in a continuous poset $P$, then there is $z \in P$ with $x \ll z \ll y$.

This enables a clear description of the Scott topology,

Theorem 1. The collection $\{\uparrow x: x \in D\}$ is a basis for the Scott topology on a continuous poset.

Definition 7. A continuous dcpo is a continuous poset which is also a dcpo. A domain is a continuous dcpo.

The next example is due to Scott[Sco70] and worth keeping in mind when we consider the analogous construction for globally hyperbolic spacetimes.

Example 1. The collection of compact intervals of the real line

$$
\mathbf{I} \mathbb{R}=\{[a, b]: a, b \in \mathbb{R} \& a \leq b\}
$$

ordered under reverse inclusion

$$
[a, b] \sqsubseteq[c, d] \Leftrightarrow[c, d] \subseteq[a, b]
$$

is an $\omega$-continuous dcpo: 
- $\quad$ For directed $S \subseteq \mathbf{I} \mathbb{R}, \bigsqcup S=\bigcap S$,

- $I \ll J \Leftrightarrow J \subseteq \operatorname{int}(I)$, and

- $\{[p, q]: p, q \in \mathbb{Q} \& p \leq q\}$ is a countable basis for $\mathbf{I} \mathbb{R}$.

The domain $\mathbf{I} \mathbb{R}$ is called the interval domain.

We also have $\max (\mathbf{I} \mathbb{R}) \simeq \mathbb{R}$ in the Scott topology. Approximation can help explain why:

Example 2. A basic Scott open set in $\mathbf{I} \mathbb{R}$ is

$$
\uparrow[a, b]=\{x \in \mathbf{I} \mathbb{R}: x \subseteq(a, b)\} .
$$

One of the interesting things about $\mathbf{I} \mathbb{R}$ is that it is a domain that is derived from an underlying poset with an abundance of order theoretic structure. Part of this structure is that the real line is bicontinuous, a fundamental notion in the present work:

Definition 8. A continuous poset $(P, \leq)$ is bicontinuous if

- For all $x, y \in P, x \ll y$ iff for all filtered $S \subseteq P$ with an infimum,

$$
\bigwedge S \leq x \Rightarrow(\exists s \in S) s \leq y,
$$

and

- For each $x \in P$, the set $\uparrow x$ is filtered with infimum $x$.

Example 3. $\mathbb{R}, \mathbb{Q}$ are bicontinuous.

Definition 9. On a bicontinuous poset $P$, sets of the form

$$
(a, b):=\{x \in P: a \ll x \ll b\}
$$

form a basis for a topology called the interval topology.

The proof uses interpolation and bicontinuity. In contrast to a domain, a bicontinuous poset $P$ has $\uparrow x \neq \emptyset$ for each $x$, so it is rarely a dcpo. We tend to prefer the notation $\leq$ for the order on a poset that is known to be bicontinuous. Otherwise, we use the notation $\sqsubseteq$.

Definition 10. For $x, y$ in a poset $(P, \leq)$,

$$
x<y \equiv x \leq y \& x \neq y .
$$

In general, $<$ and $\ll$ are completely different ideas. 


\section{The causal structure of spacetime}

A manifold $\mathcal{M}$ is a locally Euclidean Hausdorff space that is connected and has a countable basis. Such spaces are paracompact. A Lorentz metric on a manifold is a symmetric, nondegenerate tensor field of type $(0,2)$ whose signature is $(-+++)$.

Definition 11. A spacetime is a real four-dimensional ${ }^{1}$ smooth manifold $\mathcal{M}$ with a Lorentz metric $g_{a b}$.

Let $\left(\mathcal{M}, g_{a b}\right)$ be a time-orientable spacetime. Let $\Pi_{\leq}^{+}$denote the future directed causal curves, and $\Pi_{\ll}^{+}$denote the future directed time-like curves.

Definition 12. For $p \in \mathcal{M}$,

$$
I^{+}(p):=\left\{q \in \mathcal{M}:\left(\exists \pi \in \Pi_{\ll}^{+}\right) \pi(0)=p, \pi(1)=q\right\}
$$

and

$$
J^{+}(p):=\left\{q \in \mathcal{M}:\left(\exists \pi \in \Pi_{\leq}^{+}\right) \pi(0)=p, \pi(1)=q\right\}
$$

Similarly, we define $I^{-}(p)$ and $J^{-}(p)$.

We write the relation $J^{+}$as

$$
p \leq q \equiv q \in J^{+}(p) .
$$

The following properties from [HE73] are very useful:

Proposition 2. Let $p, q, r \in \mathcal{M}$. Then

(i) The sets $I^{+}(p)$ and $I^{-}(p)$ are open.

(ii) $p \leq q$ and $r \in I^{+}(q) \Rightarrow r \in I^{+}(p)$

(iii) $q \in I^{+}(p)$ and $q \leq r \Rightarrow r \in I^{+}(p)$

(iv) $\mathrm{Cl}\left(I^{+}(p)\right)=\mathrm{Cl}\left(J^{+}(p)\right)$ and $\mathrm{Cl}\left(I^{-}(p)\right)=\mathrm{Cl}\left(J^{-}(p)\right)$.

We always assume the chronology conditions that ensure $(\mathcal{M}, \leq)$ is a partially ordered set. We also assume strong causality which can be characterized as follows [Pen72]:

Theorem 2. A spacetime $\mathcal{M}$ is strongly causal iff its Alexandroff topology is Hausdorff iff its Alexandroff topology is the manifold topology.

The Alexandroff topology on a spacetime has $\left\{I^{+}(p) \cap I^{-}(q): p, q \in \mathcal{M}\right\}$ as a basis $[\mathrm{Pen} 72]^{2}$.

${ }^{1}$ The results in the present paper work for any dimension $n \geq 2$ [J93].

2 This terminology is common among relativists but order theorists use the phrase "Alexandrov topology" to mean something else: the topology generated by the upper sets. 


\section{Global hyperbolicity}

Penrose has called globally hyperbolic spacetimes "the physically reasonable spacetimes [Wal84]."

Definition 13. A spacetime $\mathcal{M}$ is globally hyperbolic if it is strongly causal and if $\uparrow a \cap \downarrow b$ is compact in the manifold topology, for all $a, b \in \mathcal{M}$.

Theorem 3 ([KP]). If $\mathcal{M}$ is globally hyperbolic, then $(\mathcal{M}, \leq)$ is a bicontinuous poset with $\ll=I^{+}$whose interval topology is the manifold topology.

This result motivates the following definition:

Definition 14. A poset $(X, \leq)$ is globally hyperbolic if it is bicontinuous and each interval $[a, b]=\{x: a \leq x \leq b\}$ is compact in the interval topology.

Globally hyperbolic posets have rich enough structure that we can deduce many properties of spacetime from them without appealing to differentiable structure or geometry. Here is one such example:

Definition 15. Let $(X, \leq)$ be a globally hyperbolic poset. A subset $\pi \subseteq X$ is a causal curve if it is compact, connected and linearly ordered. We define

$$
\pi(0):=\perp \text { and } \pi(1):=\top
$$

where $\perp$ and $\top$ are the least and greatest elements of $\pi$. For $P, Q \subseteq X$,

$$
C(P, Q):=\{\pi: \pi \text { causal curve, } \pi(0) \in P, \pi(1) \in Q\}
$$

and call this the space of causal curves between $P$ and $Q$.

This definition is motivated by the fact that a subset of a globally hyperbolic spacetime $\mathcal{M}$ is the image of a causal curve iff it is the image of a continuous monotone increasing $\pi:[0,1] \rightarrow \mathcal{M}$ iff it is a compact connected linearly ordered subset of $(\mathcal{M}, \leq)$.

Theorem 4 ([Mar06]). If $(X, \leq)$ is a separable globally hyperbolic poset, then the space of causal curves $C(P, Q)$ is compact in the Vietoris topology and hence in the upper topology.

This result plays an important role in the proofs of certain singularity theorems [Wal84], in establishing the existence of maximum length geodesics [HE73], and in the proof of certain positive mass theorems [Pen93]. Moreover, while events in spacetime are maximal elements of $\mathbf{I} \mathcal{M}$, causal curves are maximal elements in a higher order domain $\mathrm{C}(\mathbf{I} \mathcal{M})$, called the convex powerdomain of $\mathbf{I} \mathcal{M}$. This is discussed in more detail in [Mar06].

We can also deduce new aspects of spacetime. Globally hyperbolic posets are very much like the real line. In fact, a well-known domain theoretic construction pertaining to the real line extends in perfect form to the globally hyperbolic posets: 
Theorem 5 ([KP]). The closed intervals of a globally hyperbolic poset $X$

$$
\mathbf{I} X:=\{[a, b]: a \leq b \& a, b \in X\}
$$

ordered by reverse inclusion

$$
[a, b] \sqsubseteq[c, d] \equiv[c, d] \subseteq[a, b]
$$

form a continuous domain with

$$
[a, b] \ll[c, d] \equiv a \ll c \& d \ll b .
$$

The poset $X$ has a countable basis iff $\mathbf{I} X$ is $\omega$-continuous. Finally,

$$
\max (\mathbf{I} X) \simeq X
$$

where the set of maximal elements has the relative Scott topology from $\mathbf{I} X$.

This observation - that spacetime has a canonical domain theoretic model - teaches us something new: from only a countable set of events and the causality relation, one can reconstruct spacetime in a purely order theoretic manner. Explaining this requires domain theory.

\section{Spacetime from a discrete causal set}

An abstract basis is a set $(C, \ll)$ with a transitive relation that is interpolative from the - direction:

$$
F \ll x \Rightarrow(\exists y \in C) F \ll y \ll x,
$$

for all finite subsets $F \subseteq C$ and all $x \in F$. Suppose, though, that it is also interpolative from the + direction:

$$
x \ll F \Rightarrow(\exists y \in C) x \ll y \ll F .
$$

Then we can define a new abstract basis of intervals

$$
\operatorname{int}(C)=\{(a, b): a \ll b\}=\ll \subseteq C^{2}
$$

whose relation is

$$
(a, b) \ll(c, d) \equiv a \ll c \& d \ll b .
$$

Let $\mathbf{I} C$ denote the ideal completion of the abstract basis $\operatorname{int}(C)$.

Theorem $6([\mathbf{K P}])$. Let $C$ be a countable dense subset of a globally hyperbolic spacetime $\mathcal{M}$ and $\ll=I^{+}$be timelike causality. Then

$$
\max (\mathbf{I} C) \simeq \mathcal{M}
$$

where the set of maximal elements have the Scott topology. 
In "ordering the order" $I^{+}$, taking its completion, and then the set of maximal elements, we recover spacetime by reasoning only about the causal relationships between a countable dense set of events. One objection to this might be that we begin from a dense set $C$, and then order theoretically recover the space $\mathcal{M}$ - but dense is a topological idea so we need to know the topology of $\mathcal{M}$ before we can recover it! But the denseness of $C$ can be expressed in purely causal terms:

$$
C \text { dense } \equiv(\forall x, y \in \mathcal{M})(\exists z \in C) x \ll z \ll y .
$$

Now the objection might be that we still have to reference $\mathcal{M}$. We too would like to not reference $\mathcal{M}$ at all. However, some global property needs to be assumed, either directly or indirectly, in order to reconstruct $\mathcal{M}$.

Theorem 6 is very different from results like "Let $\mathcal{M}$ be a certain spacetime with relation $\leq$. Then the interval topology is the manifold topology." Here we identify, in abstract terms, a process by which a countable set with a causality relation determines a space. The process is entirely order theoretic in nature, spacetime is not required to understand or execute it (i.e., if we put $C=\mathbb{Q}$ and $\ll=<$, then $\max (\mathbf{I} C) \simeq \mathbb{R})$. In this sense, our understanding of the relation between causality and the topology of spacetime is now explainable independently of geometry.

Ideally, one would now like to know what constraints on $C$ in general imply that $\max (\mathbf{I} C)$ is a manifold.

\section{Spacetime as a domain}

The category of globally hyperbolic posets is naturally isomorphic to a special category of domains called interval domains.

Definition 16. An interval poset is a poset $D$ that has two functions left : $D \rightarrow \max (D)$ and right $: D \rightarrow \max (D)$ such that

(i) Each $x \in D$ is an "interval" with $\operatorname{left}(x)$ and right $(x)$ as endpoints:

$$
(\forall x \in D) x=\operatorname{left}(x) \sqcap \operatorname{right}(x),
$$

(ii) The union of two intervals with a common endpoint is another interval: For all $x, y \in D$, if $\operatorname{right}(x)=\operatorname{left}(y)$, then

$$
\operatorname{left}(x \sqcap y)=\operatorname{left}(x) \quad \& \operatorname{right}(x \sqcap y)=\operatorname{right}(y),
$$

(iii) Each point $p \in \uparrow x \cap \max (D)$ of an interval $x \in D$ determines two subintervals, $\operatorname{left}(x) \sqcap p$ and $p \sqcap \operatorname{right}(x)$, with endpoints:

$$
\begin{array}{lll}
\operatorname{left}(\operatorname{left}(x) \sqcap p)=\operatorname{left}(x) & \& & \operatorname{right}(\operatorname{left}(x) \sqcap p)=p \\
\operatorname{left}(p \sqcap \operatorname{right}(x))=p & \& & \operatorname{right}(p \sqcap \operatorname{right}(x))=\operatorname{right}(x)
\end{array}
$$


Notice that a nonempty interval poset $D$ has $\max (D) \neq \emptyset$ by definition. With interval posets, we only assume that infima indicated in the definition exist; in particular, we do not assume the existence of all binary infima.

Definition 17. For an interval poset $(D$, left, right $)$, the relation $\leq$ on $\max (D)$ is

$$
a \leq b \equiv(\exists x \in D) a=\operatorname{left}(x) \& b=\operatorname{right}(x)
$$

for $a, b \in \max (D)$.

The axioms of interval posets imply that $(\max (D), \leq)$ is a poset.

Definition 18. An interval domain is an interval poset ( $D$, left, right) where $D$ is a continuous dcpo such that

(i) If $p \in \uparrow x \cap \max (D)$, then

$$
\uparrow(\operatorname{left}(x) \sqcap p) \neq \emptyset \quad \& \quad \uparrow(p \sqcap \operatorname{right}(x)) \neq \emptyset .
$$

(ii) For all $x \in D$, the following are equivalent:

(a) $\uparrow x \neq \emptyset$

(b) $(\forall y \in[\operatorname{left}(x), \cdot])(y \sqsubseteq x \Rightarrow y \ll \operatorname{right}(y)$ in $[\cdot, \operatorname{right}(y)])$

(c) $(\forall y \in[\cdot, \operatorname{right}(x)])(y \sqsubseteq x \Rightarrow y \ll \operatorname{left}(y) \quad$ in $[\operatorname{left}(y), \cdot])$

(iii) Invariance of endpoints under suprema:

(a) For all directed $S \subseteq[p, \cdot]$

$$
\operatorname{left}(\bigsqcup S)=p \quad \& \quad \operatorname{right}(\bigsqcup S)=\operatorname{right}(\bigsqcup T)
$$

for any directed $T \subseteq[q, \cdot]$ with $\operatorname{right}(T)=\operatorname{right}(S)$.

(b) For all directed $S \subseteq[\cdot, q]$

$$
\operatorname{left}(\bigsqcup S)=\operatorname{left}(\bigsqcup T) \quad \& \quad \operatorname{right}(\bigsqcup S)=q
$$

for any directed $T \subseteq[\cdot, p]$ with $\operatorname{left}(T)=\operatorname{left}(S)$.

(iv) Intervals are compact: For all $x \in D, \uparrow x \cap \max (D)$ is Scott compact.

Interval domains are interval posets whose axioms also take into account the completeness and approximation present in a domain: (i) says if a point $p$ belongs to the interior of an interval $x \in D$, the subintervals left $(x) \sqcap p$ and $p \sqcap \operatorname{right}(x)$ both have nonempty interior; (ii) says an interval has nonempty interior iff all intervals that contain it have nonempty interior locally; (iii) explains the behavior of endpoints when taking suprema.

For a globally hyperbolic $(X, \leq)$, we define:

$$
\text { left }: \mathbf{I} X \rightarrow \mathbf{I} X::[a, b] \mapsto[a]
$$

and

$$
\text { right }: \mathbf{I} X \rightarrow \mathbf{I} X::[a, b] \mapsto[b] .
$$


Lemma 1. If $(X, \leq)$ is a globally hyperbolic poset, then (IX, left, right) is an interval domain.

In essence, this is the only example.

Lemma 2. If ( $D$, left, right) is an interval domain, then $(\max (D), \leq)$ is a globally hyperbolic poset.

The equivalence between globally hyperbolic posets and interval domains is as follows:

Definition 19. The category IN of interval domains and commutative maps is given by

- objects Interval domains ( $D$, left, right).

- arrows Scott continuous $f: D \rightarrow E$ that commute with left and right, i.e., such that both
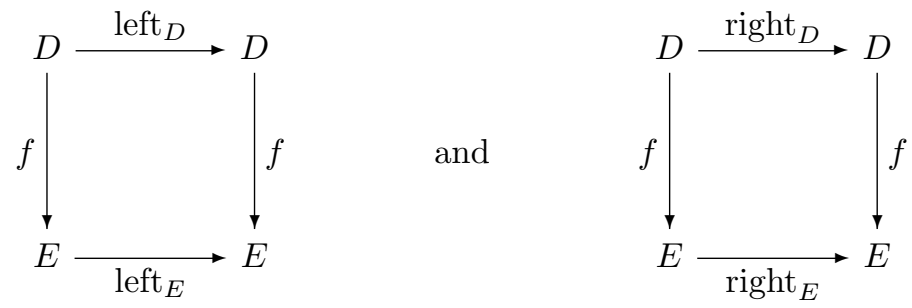

commute.

- identity $1: D \rightarrow D$.

- composition $f \circ g$.

Definition 20. The category $\underline{G}$ is given by

- objects Globally hyperbolic posets $(X, \leq)$.

- arrows Continuous in the interval topology, monotone.

- identity $1: X \rightarrow X$.

- composition $f \circ g$.

It is routine to verify that $\underline{\mathrm{IN}}$ and $\underline{\mathrm{G}}$ are categories.

Proposition 3. The correspondence $\mathbf{I}: \underline{\mathrm{G}} \rightarrow \underline{\mathrm{IN}}$ given by

$$
\begin{gathered}
(X, \leq) \mapsto(\mathbf{I} X, \text { left, right }) \\
(f: X \rightarrow Y) \mapsto(\bar{f}: \mathbf{I} X \rightarrow \mathbf{I} Y)
\end{gathered}
$$

is a functor between categories.

Proposition 4. The correspondence $\max : \underline{\mathrm{IN}} \rightarrow \underline{\mathrm{G}}$ given by

$$
\begin{gathered}
(D, \text { left, right }) \mapsto(\max (D), \leq) \\
(f: D \rightarrow E) \mapsto\left(\left.f\right|_{\max (D)}: \max (D) \rightarrow \max (E)\right)
\end{gathered}
$$

is a functor between categories. 
Before the statement of the main theorem in this section, we recall the definition of a natural isomorphism.

Definition 21. A natural transformation $\eta: F \rightarrow G$ between functors $F$ : $\mathcal{C} \rightarrow \mathcal{D}$ and $G: \mathcal{C} \rightarrow \mathcal{D}$ is a collection of arrows $\left(\eta_{X}: F(X) \rightarrow G(X)\right)_{X \in \mathcal{C}}$ such that for any arrow $f: A \rightarrow B$ in $\mathcal{C}$,

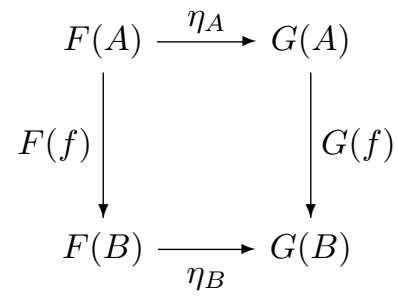

commutes. If each $\eta_{X}$ is an isomorphism, $\eta$ is a natural isomorphism.

Categories $\mathcal{C}$ and $\mathcal{D}$ are equivalent when there are functors $F: \mathcal{C} \rightarrow \mathcal{D}$ and $G: \mathcal{D} \rightarrow \mathcal{C}$ and natural isomorphisms $\eta: 1_{\mathcal{C}} \rightarrow G F$ and $\mu: 1_{\mathcal{D}} \rightarrow F G$.

Theorem 7 ([KP]). The category of globally hyperbolic posets is equivalent to the category of interval domains.

This result suggests that questions about spacetime can be converted to domain theoretic form, where we can use domain theory to answer them, and then translate the answers back to the language of physics (and viceversa). Notice too that the category of interval posets and commutative maps is equivalent to the category of posets and monotone maps.

It also shows that causality between events is equivalent to an order on regions of spacetime. Most importantly, we have shown that globally hyperbolic spacetime with causality is equivalent to a structure $\mathbf{I} X$ whose origins are "discrete." This is the formal explanation for why spacetime can be reconstructed from a countable dense set of events in a purely order theoretic manner.

\section{Time and measurement}

A domain is a partially ordered set with intrinsic notions of completeness and approximation defined by the order. A measurement is a function $\mu$ that to each informative object $x$ assigns a number $\mu x$ which measures the information content of the object $x$. Let us now define the latter term precisely before discussing it further.

A function $f: D \rightarrow E$ between domains is $S$ cott continuous if the inverse image of a Scott open set in $E$ is Scott open in $D$. This is equivalent [AJ94] to saying that $f$ is monotone, 


$$
(\forall x, y \in D) x \sqsubseteq y \Rightarrow f(x) \sqsubseteq f(y),
$$

and that it preserves directed suprema:

$$
f(\bigsqcup S)=\bigsqcup f(S),
$$

for all directed $S \subseteq D$. In particular, for the domain $[0, \infty)^{*}$ of nonnegative reals in their opposite order, a Scott continuous function $\mu: D \rightarrow[0, \infty)^{*}$ will satisfy

1. For all $x, y \in D, x \sqsubseteq y \Rightarrow \mu x \geq \mu y$, and

2. If $\left(x_{n}\right)$ is an increasing sequence in $D$, then

$$
\mu\left(\bigsqcup_{n \geq 1} x_{n}\right)=\lim _{n \rightarrow \infty} \mu x_{n} .
$$

This is the case of Scott continuity that we are most interested in presently:

Definition 22. A Scott continuous $\mu: D \rightarrow[0, \infty)^{*}$ is said to measure the content of $x \in D$ if for all Scott open sets $U \subseteq D$,

$$
x \in U \Rightarrow(\exists \varepsilon>0) x \in \mu_{\varepsilon}(x) \subseteq U
$$

where

$$
\mu_{\varepsilon}(x):=\{y \in D: y \sqsubseteq x \&|\mu x-\mu y|<\varepsilon\}
$$

are called the $\varepsilon$-approximations of $x$.

We often refer to $\mu$ as simply 'measuring' $x \in D$ or as measuring $X \subseteq D$ when it measures each element of $X$. The last definition, as well as the next, easily extend to maps $\mu$ that take values in an arbitrary domain $E$.

Definition 23. A measurement $\mu: D \rightarrow[0, \infty)^{*}$ is a Scott continuous map that measures the content of $\operatorname{ker}(\mu):=\{x \in D: \mu x=0\}$.

The order on a domain $D$ defines a clear sense in which one object has 'more information' than another: a qualitative view of information content. The definition of measurement attempts to identify those monotone mappings $\mu$ which offer a quantitative measure of information content in the sense specified by the order. The essential point in the definition of measurement is that $\mu$ measure content in a manner that is consistent with the particular view offered by the order. There are plenty of monotone mappings that are not measurements - and while some of them may measure information content in some other sense, each sense must first be specified by a different information order. The definition of measurement is then a minimal test that a function $\mu$ must pass if we are to regard it as providing a measure of information content.

We now consider a few properties that measures of information content have which arbitrary monotone mappings in general need not have: qualities that make them 'different' from maps that are simply monotone. Other such properties may be found in [Mar00a]. 
Theorem 8 ([Mar00a]). Let $\mu: D \rightarrow[0, \infty)^{*}$ be a measurement.

(i) If $x \in \operatorname{ker}(\mu)$, then $x \in \max (D)=\{x \in D: \uparrow x=\{x\}\}$.

(ii) If $\mu$ measures the content of $y \in D$, then

$$
(\forall x \in D) x \sqsubseteq y \& \mu x=\mu y \Rightarrow x=y .
$$

(iii) If $\mu$ measures $X \subseteq D$, then

$$
\left\{\uparrow \mu_{\varepsilon}(x) \cap X: x \in X, \varepsilon>0\right\}
$$

is a basis for the Scott topology on $X$.

A global time function $t: \mathcal{M} \rightarrow \mathbb{R}$ on a globally hyperbolic spacetime $\mathcal{M}$ is a continuous function such that $x<y \Rightarrow t(x)<t(y)$ and $t^{-1}(r)=\Sigma$ is a Cauchy surface for $\mathcal{M}$, for each $r \in \mathbb{R}$.

Theorem 9. For any global time function $t: \mathcal{M} \rightarrow \mathbb{R}$ on a globally hyperbolic spacetime, the function $\Delta t: \mathcal{M} \rightarrow[0, \infty)^{*}$ given by $\Delta t[a, b]=t(b)-t(a)$ measures all of $\mathbf{I}(\mathcal{M})$. It is a measurement with $\operatorname{ker}(\Delta t)=\max (\mathbf{I}(\mathcal{M}))$.

Let $d: \mathbf{I}(\mathcal{M}) \rightarrow[0, \infty)^{*}$ denote the Lorentz distance on a globally hyperbolic spacetime

$$
d[a, b]=\sup _{\pi_{a b}} \operatorname{len}\left(\pi_{a b}\right)
$$

where the sup is taken over all causal curves that join $a$ to $b$.

Definition 24. The interval topology on a continuous poset $P$ exists when sets of the form

$$
(a, b)=\{x \in P: a \ll x \ll b\} \quad \& \uparrow x=\{y \in P: x \ll y\}
$$

form a basis for a topology on $P$.

For bicontinuous posets, this definition of interval topology is equivalent to the definition considered earlier. A function between continuous posets is interval continuous when each poset has an interval topology and the inverse image of an interval open set is interval open. By the bicontinuity of $\mathcal{M}$, the interval topology on $\mathbf{I}(\mathcal{M})$ exists, so we can consider interval continuity for functions $\mathbf{I}(\mathcal{M}) \rightarrow[0, \infty)^{*}$.

Theorem 10. The Lorentz distance $d: \mathbf{I}(\mathcal{M}) \rightarrow[0, \infty)^{*}$ has the following properties:

(i) It is monotone: $x \leq y \Rightarrow d(x) \geq d(y)$,

(ii) It preserves the way below relation: $x \ll y \Rightarrow d(x)>d(y)$,

(iii) It is interval continuous and hence Scott continuous.

It does not measure $\mathbf{I}(\mathcal{M})$ at any point of $\operatorname{ker}(d)$. 
That the Lorentz distance is not a measurement is a direct consequence of the fact that a clock travelling at the speed of light records no time as having elapsed i.e. the set of null intervals is equal to

$$
\operatorname{ker}(d) \backslash \max (\mathbf{I}(\mathcal{M})) \neq \emptyset
$$

but measurements always have the property that $\mu x=0$ implies $x \in \max (D)$ (Theorem 8).

In fact, no interval continuous function $\mu: \mathbf{I}(\mathcal{M}) \rightarrow[0, \infty)^{*}$ can be a measurement: by interval continuity, $\mu x=0$ for any $x$ with $\uparrow x=\emptyset$. Just like the Lorentz distance, an interval continuous $\mu$ will also assign 0 to "null intervals." In this way, we see that interval continuity captures an essential aspect of the Lorentz distance. In addition, since $\Delta t$ is a measurement, it cannot be interval continuous. This provides a surprising topological distinction between the Newtonian and relativistic concepts of time: $d$ is interval continuous, $\Delta t$ is not. Put another way, $\Delta t$ can be used to reconstruct the topology of spacetime (Theorem 8(iii)), while $d$ is used to reconstruct its geometry.

\section{Spacetime geometry from a discrete causal set}

Let us return now to the reconstruction of spacetime (Section 5) from a countable dense set $(C, \ll)$. Specifically, we take the rounded ideal completion $\mathbf{I}(C)$ of the abstract basis of intervals

$$
\operatorname{int}(C)=\{(a, b): a \ll b\}=\ll \subseteq C^{2}
$$

whose relation is

$$
(a, b) \ll(c, d) \equiv a \ll c \& d \ll b .
$$

We are then able to recover spacetime as

$$
\max (\mathbf{I} C) \simeq \mathcal{M}
$$

where the set of maximal elements have the Scott topology. Let us now suppose that in addition to $\operatorname{int}(C)$ that we also begin with a countable collection of numbers $l_{a b}$ chosen for each $(a, b) \in \operatorname{int}(C)$ in such a way that the map

$$
\operatorname{int}(C) \rightarrow[0, \infty)^{*}::(a, b) \mapsto l_{a b}
$$

is monotone. Then in the process of reconstructing spacetime, we can also construct the Scott continuous function $d: \mathbf{I} C \rightarrow[0, \infty)^{*}$ given by

$$
d(x)=\inf \left\{l_{a b}:(a, b) \ll x\right\} .
$$

In the event that the countable number of $l_{a b}$ chosen are the Lorentz distances $l_{a b}=d[a, b]$, then the function $d$ constructed above yields the Lorentz distance for any spacetime interval, the reason being that both are Scott continuous and are equal on a basis of the domain.

Thus, from a countable dense set of events and a countable set of distances, we can reconstruct the spacetime manifold together with its geometry in a purely order theoretic manner. 


\section{Conclusions}

We have seen the following ideas in this paper:

1. how to reconstruct the spacetime topology from the causal structure using purely order-theoretic ideas,

2. an abstract order-theoretic definition of global hyperbolicity,

3. that one can reconstruct spacetime, meaning its topology and geometry, from a countable dense subset,

4. an equivalence of categories between the category of interval domains and the category of globally hyperbolic posets.

5. a topological distinction between Newtonian and relativistic notions of time.

\section{References}

[AJ94] S. Abramsky and A. Jung. Domain theory. In T. S. E. Maibaum S. Abramsky, D. M. Gabbay, editor, Handbook of Logic in Computer Science, vol. III. Oxford University Press, 1994.

[Beem] John K. Beem, Paul E. Ehrlich and Kevin L. Easley. Global Lorentzian Geometry. CRC Press, 1996.

[Cha31] S. Chandrasekhar. The maximum mass of ideal white dwarfs. Astrophysical Journal, 74:81-82, 1931.

[Faj00] Lisbeth Fajstrup. Loops, ditopology and deadlocks: Geometry and concurrency. Math. Structures Comput. Sci., 10(4):459-480, 2000.

[GFR98] Eric Goubault, Lisbeth Fajstrup, and Martin Raussen. Algebraic topology and concurrency. Department of Mathematical Sciences RR-98-2008, Aalborg University, 1998. Presented at MFCS 1998 London.

[GKK] G.Gierz, K.H.Hoffman, K.Keimel, J.D.Lawson, M.Mislove, and D.S.Scott. Continuous lattices and domains. Number 93 in Encyclopedia of Mathematics and its Applications. Cambridge University Press, 2003.

[HE73] S.W. Hawking and G.F.R. Ellis. The large scale structure of space-time. Cambridge Monographs on Mathematical Physics. Cambridge University Press, 1973.

[J93] P. S. Joshi. Global aspects in gravitation and cosmology. International Series of Monographs on Physics 87. Oxford Science Publications, 1993.

[Mal77] David Malement. The class of continuous timelike curves determines the topology of spacetime. J. Math. Phys., 18(7):1399-1404, 1977.

[Mar00a] Keye Martin. A foundation for computation. PhD thesis, Department of Mathematics, Tulane University, 2000.

[Mar00b] Keye Martin. The measurement process in domain theory. In 27th International Colloquium on Automata, Languages and Programming (ICALP'00), number 1853 in Lecture Notes In Computer Science, pages 116-126. Springer-Verlag, 2000.

[Mar06] Keye Martin. Compactness of the space of causal curves. Journal of Classical and Quantum Gravity, 2006. 
[KP] Keye Martin and Prakash Panangaden. A domain of spacetime intervals in general relativity. Communications in Mathematical Physics, 267(3):563586, November 2006.

[Pen65] Roger Penrose. Gravitational collapse and space-time singularities. Phys. Rev. Lett., 14:57-59, 1965.

[Pen72] Roger Penrose. Techniques of differential topology in relativity. Society for Industrial and Applied Mathematics, 1972.

[Pen93] R. Penrose, R.D. Sorkin and E. Woolgar. A positive mass theorem based on the focusing and retardation of null geodesics. Submitted to Commun. Math. Phys., arXiv:gr-qc/9301015, 1993.

[Sco70] Dana Scott. Outline of a mathematical theory of computation. Technical Monograph PRG-2, Oxford University Computing Laboratory, 1970.

[Sor91] R. Sorkin. Spacetime and causal sets. In J. D'Olivo et. al., editor, Relativity and Gravitation: Classical and Quantum. World Scientific, 1991.

[SW96] R.D. Sorkin and E. Woolgar. A causal order for spacetimes with $c^{0}$ Lorentzian metrics: Proof of compactness of the space of causal curves. Classical and Quantum Gravity, 13:1971-1994, 1996.

[Wal84] R.M. Wald. General relativity. The University of Chicago Press, 1984. 